\title{
Disorder-Induced Stabilization of the Pseudogap in Strongly Correlated Systems
}

\author{
Simone Chiesa * Prabuddha B. Chakraborty, Warren E. Pickett, and Richard T. Scalettar \\ Dept. of Physics, University of California, Davis, CA 95616
}

\begin{abstract}
The interplay of strong interaction and strong disorder, as contained in the Anderson-Hubbard model, is addressed using two non-perturbative numerical methods: the Lanczos algorithm in the grand canonical ensemble at zero temperature and Quantum Monte Carlo. We find distinctive evidence for a zero-energy anomaly which is robust upon variation of doping, disorder and interaction strength. Its similarities to, and differences from, pseudogap formation in other contexts, including perturbative treatments of interactions and disorder, classical theories of localized charges, and in the clean Hubbard model, are discussed.
\end{abstract}

"Pseudogap" anomalies in the single particle density of states are a central feature of seemingly disparate materials and models. On the one hand, they have an early history in the metal-insulator transition and the study of the interplay between disorder and inter-particle interaction. In the metallic limit of weak coupling and weak disorder, Altshuler and Aronov (AA) showed 1], by means of perturbation theory, that there is a depression of the spectral density at the chemical potential, the magnitude of the depression being dependent on the interaction strength. In the opposite limit of completely localized charges (where the model becomes classical), Efros and Shklovskii (ES) 2] have shown that the combined effect of the unscreened Coulomb potential and disorder also gives rise to an anomaly at the chemical potential the Coulomb gap.

On the other hand, in contrast to this situation in which randomness and electron-electron correlation both are crucial, pseudogap anomalies also arise in cuprate superconductors and the Hubbard model with no disorder. While particle density does not play a central role in either the AA and ES pseudogaps, in the high $T_{c}$ materials the pseudogap is confined to a low-doping region between the superconducting dome and the parent antiferromagnetic material. Likewise, in numerical studies of the Hubbard Hamiltonian [3, 4, 5] the pseudogap is absent for particle densities $\rho<0.80$.

That disorder is essential to the pseudogap in one situation, yet present in the clean system in another, raises a fundamental question: What role does randomness play in low energy anomalies in the density of states of strongly correlated systems? In this manuscript, we suggest that randomness stabilizes the pseudogap. Indeed, we demonstrate two remarkable features of the pseudogap in the disordered Hubbard model. First, the density of states anomaly persists in the limit of an infinitely repulsive local potential $U$, even though the magnetic energy scale $J \propto t^{2} / U$ is driven to zero. Second, it is independent of doping for a wide range of disorder and interaction strengths. The insensitivity of the pseudogap to doping is a novel effect related to the presence of disorder that has been observed experimentally [] $]$.
We consider the Anderson-Hubbard Hamiltonian,

$$
H\left(\left\{\epsilon_{i}\right\}\right)=-t \sum_{i j \sigma}^{\prime} c_{i \sigma}^{\dagger} c_{j \sigma}+\sum_{i \sigma} \epsilon_{i} n_{i \sigma}+|U| \sum_{i} n_{i \uparrow} n_{i \downarrow}
$$

where the on-site energies $\epsilon_{i}$ are sampled uniformly from the interval $[-\Delta / 2: \Delta / 2], c_{i \sigma}^{\dagger}\left(c_{i \sigma}\right)$ are fermion creation(annihilation) operators for site $i$ and spin $\sigma$, and $n_{i \sigma}=c_{i \sigma}^{\dagger} c_{i \sigma}$. The primed summation is on nearest neighbors only. We diagonalize this Hamiltonian on 10-site square clusters using the Lanczos algorithm at $T=0$ and on larger, 64-site clusters using finite temperature determinant Quantum Monte Carlo (DQMC). As usual, physical properties are computed as averages over many disorder realizations.

Since both computational methods are well described in a number of previous publications [7, 8] we focus our technical discussion only on the use of the grandcanonical ensemble to carry out the averaging process. In the DQMC case this is accomplished naturally as this method works, by construction, directly within a grand-canonical scheme. The Lanczos method, on the other hand, is a canonical technique that diagonalizes the Hamiltonian in sectors of the Hilbert space with constant number of particles. Since we are interested in ground state properties, a given choice of chemical potential $\mu$ has the effect of singling out that particle number sector whose ground state minimizes $\langle H-\mu N\rangle$. Which particular sector is selected is, of course, dependent on the disorder realization defining $H\left(\left\{\epsilon_{i}\right\}\right)$ and it is not known a priori. Although this requires diagonalization of all particle sectors it also allows the treatment of non-commensurate fillings. Since the size of the system is fairly small we take advantage of the possibility of changing the boundary conditions of the electronic wave function to reduce finite size errors. The second quantized Hamiltonian becomes therefore a function of $k$, a vector belonging to the first Brillouin zone of the simulation lattice. Within the grand-canonical ensemble $k$ is treated as a quenched disorder variable and sampled uniformly. This is found to be important in the calculation of the spectral density for the model considered here. In the limit of vanishing disorder this scheme reduces to the integration over boundary condition technique [9, 10]. With no disorder 


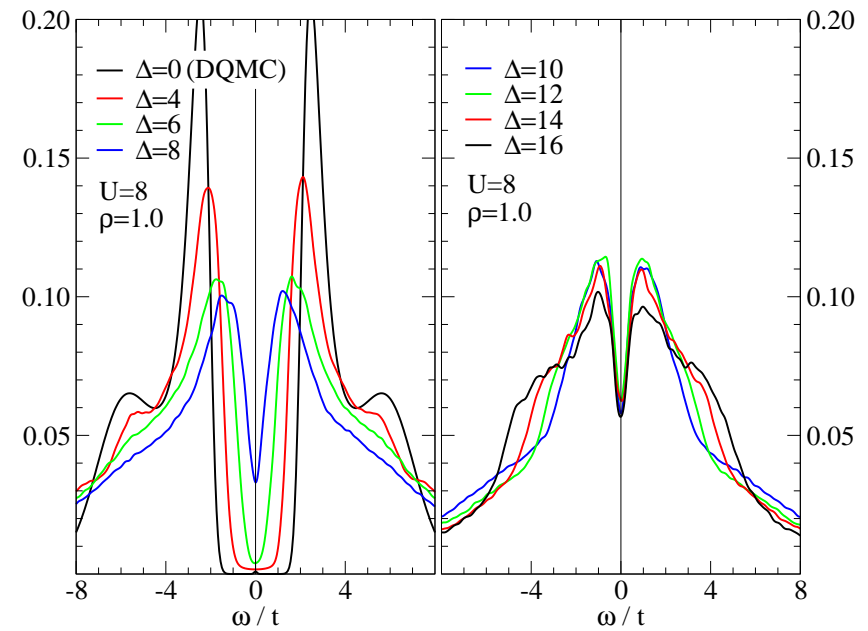

FIG. 1: Evolution of the local spectral density, $A(\omega)$, at $\rho=1$ for $U=8$ as a function of $\Delta$. Left panel: $\Delta \leq U$. The

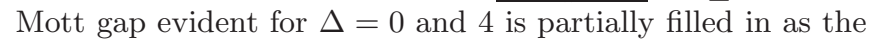
randomness increases, so that the value at the Fermi surface, $A(\omega=0)$, becomes finite at $\Delta=U$. Right panel: $\Delta>U$. A pseudogap survives even for large randomness. The spectra are the average of 1000 independent disorder realizations.

and no interaction one recovers the exact spectral density in the thermodynamic limit.

We argue that the density of states obtained on the cluster sizes used here is relevant both because of the restoration of the thermodynamic limit by boundary condition averaging, and because finite size errors become increasingly small in the strong disorder case on which we focus. It is also worth noting that, unlike studies of other phenomena like long range magnetic or superconducting order in the Hubbard Hamiltonian, which critically rely on finite size scaling on large lattices, the density of states is less sensitive to system size. The AA calculation provides a particularly clear example of this statement as the zero-energy anomaly was predicted using a class of diagrams that failed to capture localization.

The disorder-averaged spectral density is given by

$$
A(\omega)=\frac{1}{N_{s}} \sum_{i=1}^{N_{s}} \frac{1}{\Delta \Omega_{\mathrm{BZ}}} \int d k d \epsilon A_{i}\left(k,\left\{\epsilon_{i}\right\}, \omega\right)
$$

where $N_{s}$ and $\Omega_{\mathrm{BZ}}$ are, respectively, the number of sites and the volume of the Brillouin zone and the fermion addition part of $A_{i}\left(k,\left\{\epsilon_{i}\right\}, \omega\right)$ is defined as

$$
A_{i}\left(k,\left\{\epsilon_{i}\right\}, \omega\right)=-\frac{1}{\pi} \operatorname{Im}\left\langle c_{i} \frac{1}{\omega-H+E_{0}+i \eta} c_{i}^{\dagger}\right\rangle
$$

and computed using the continued fraction algorithm of Haydock et al. [11]. The electron subtraction spectrum is obtained analogously by interchanging $c_{i}$ and $c_{i}^{\dagger}$. In Eq. $3 E_{0}$ is the ground state energy of $H \equiv H\left(k,\left\{\epsilon_{i}\right\}\right)$ and $\eta$ is a small real parameter giving the broadening

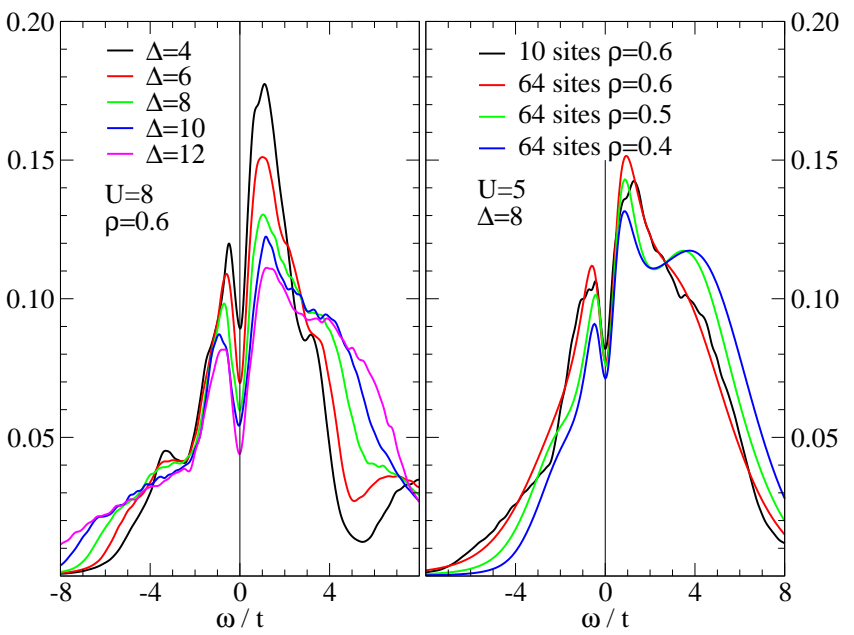

FIG. 2: Evolution of the local spectral density away from half-filling (no Mott gap at $\Delta=0$ ). Left panel: $A(\omega)$ as a function of $\Delta . \Delta$ ranges from well below $U$ to well above. The dip in $A(\omega)$ at $\omega=0$ becomes deeper as $\Delta$ increases. In the clean limit $\Delta=0$ we observe no pseudogap this far from half-filling, in agreement with [3, 4, 5]. Right panel: DQMC results on a $8 \times 8=64$ site lattice at $T=t / 6$ averaging over 64 disorder realizations. The scale (width) of the pseudogap is largely independent of doping. For $\rho=0.6$ the 10 -site Lanczos result is also reported. Despite the different cluster size the agreement between the two techniques is excellent.

of the $\delta$ functions constituting the spectrum. $A(\omega)$ is directly measured in tunneling spectroscopy, photoemission and inverse photoemission experiments. Since disorder averaging restores particle-hole symmetry, it suffices to consider the evolution of $A(\omega)$ at densities $\rho \leq 1$. In DQMC, $A(\omega)$ is obtained by a maximum entropy analytic continuation of the imaginary time Green's function 12. computed using periodic boundary conditions $(k=0)$.

There are three different independent parameters $(t$ sets the unit of energy) that we examine when analyzing the spectral densitiy: the interaction $U$, the disorder $\Delta$ and the doping $\rho$. We start by considering the dependence on $\Delta$ for the half-filled case and constant $U=8$. On increasing $\Delta$, for $\Delta<U$, the Mott gap present at $\Delta=0$ is gradually filled in, and the evolution of the spectral density follows the expected trend (see left panel of Fig. 1). For $\Delta>U$ one might expect the residual dip to disappear completely, at least at large enough disorder, but this is not what is found numerically. The right panel of Fig. 1 shows the behavior for $\Delta=10,12,14,16$ : Increasing the disorder above $\Delta=U$ leaves a residual pseudogap independent of the disorder strength. There is a sharpness in the behavior of $A(\omega)$ at small $\omega$ which suggests that the anomaly could be nonanalytic at $T=0$. However, finite size rounding prevent a precise characterization of this feature.

The appearance of a pseudogap is remarkably different from what is found using dynamical mean field theory 


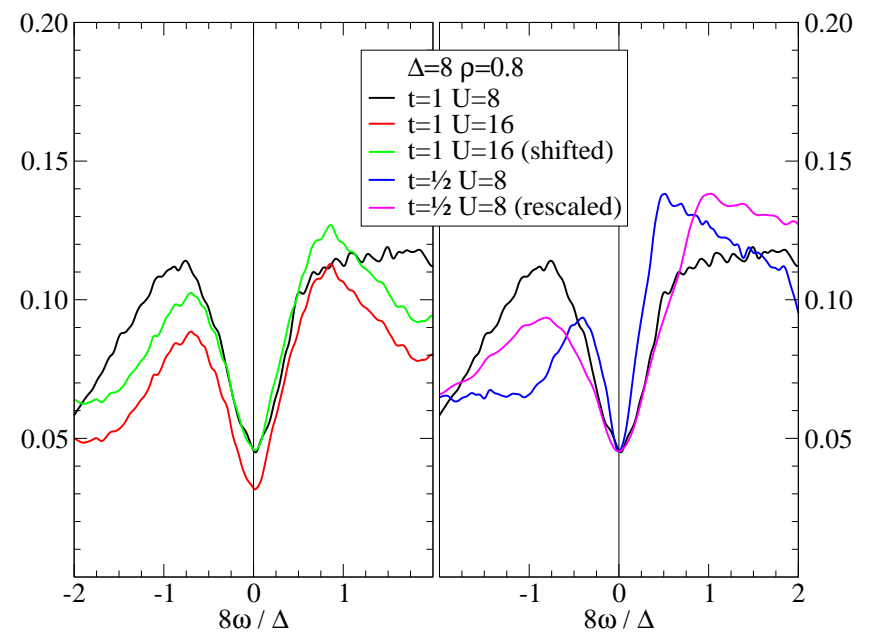

FIG. 3: Scaling of the pseudogap as a function of interaction and hopping. The key observation is that the pseudogap is unchanged when $U$ increases from $U=8$ to $U=16$ (left panel), and hence the antiferromagnetic exchange $J$ is halved, but has a width which is proportional to $t$ (right panel). The shifting in the third data set is done so as to make the two minima, for $U=8$ and $U=16$, coincide. In the last data set the energy axis was rescaled by a factor of two.

(DMFT) in a similar range of parameters 13] for the same Hamiltonian. DMFT predicts, at least in the HubbardI approximation [13], that $A(\omega)$ evolves smoothly as the energy crosses the chemical potential, with no pseudogap. This indicates that the anomaly is likely to be determined by non-local, short-ranged correlations and connected to reduced dimensionality.

Let us now move to the incommensurate filling $\rho=0.6$, where there is no Mott gap. $A(\omega)$ is plotted in Fig. 2, A pseudogap is now evident at all disorder strengths. For $\Delta>U$ it behaves as for the $\rho=1$ case and saturates for strong enough disorder. In the $\Delta<U$ regime the pseudogap gets deeper with increasing disorder but its width remains largely unchanged. In contrast, the depth of the pseudogap which develops out of the Mott phase at $\rho=1$ decreases as $\Delta$ increases 17. Such a difference is not surprising since, at $\rho=1$, there is a cross-over from a $U>\Delta$ regime, dominated by the Mott-gap scale to the $\Delta>U$ regime characterized by the pseudogap scale. On the other hand, away from half filling, the pseudogap scale is always the leading instability and continues to persist in the highly disordered regime. It remains unclear why the depth of the pseudogap saturates instead of moving monotonically to 0 as $\Delta$ increases. Certainly the behavior of the anomaly away from half-filling makes evident that the naive picture in which the effect of disorder is only to smear the spectral density is inappropriate.

We have carefully studied the doping dependence of the pseudogap, especially since in the clean model it is present only for small doping. In contrast, for $\Delta=10$ and $U=8$ the spectral density shows a nearly universal pseudogap: $A(\omega)$ for $\rho=0.6-1.0$ coincide over the entire pseudogap region. This feature persists in the $\Delta \leq U$ regime as soon as the system is sufficiently away from the Mott-gap dominated regime. Typically, at $\rho=0.9$, a distinct dip at $\omega=0$ is seen at all disorder strengths considered in this work. At densities $\rho \leq 0.6$ and $U \leq 5$ the sign problem in the DQMC method is mild and does not prohibit obtaining accurate spectral functions [18]. The agreement between the two numerical techniques at $\rho=0.6$ and $\Delta=8$ (see right panel of Fig 2) is excellent and gives a firmer basis to our speculations on the irrelevance of the cluster size that was exactly diagonalized. DQMC results from $\rho=0.6$ to $\rho=0.4$ also confirms the stability of the pseudogap under doping. That strong disorder can stabilize the pseudogap was also observed experimentally [6] in a conductivity study of YBCO samples where $\mathrm{Cu}$ was substituted with $\mathrm{Zn}$. The $T-\rho$ phase diagram of this heavily disordered system shows a flat pseudogap crossover line in agreement with our finding of a doping-independent pseudogap energy scale.

That variation in $\Delta$ or $\rho$ both leave the anomaly unchanged can be understood as follows. Consider, for instance, the half-filled case with $t=0$ and $\Delta>U$ in which a finite fraction of the sites remains empty. When $t \neq 0$, electrons lower their energy by delocalizing on neighboring empty sites, regardless of the overall density, as the physics is primarily local. The effect of doping does not alter this situation but merely shifts the "action" on the new set of sites lying close to $\mu$. Although the details of the effect of $t$ are ultimately responsible for the formation of the pseudogap, one can see that if the pseudogap forms and is stable upon variation in disorder then it follows that the same anomaly forms and is stable upon variation of doping.

The distinctive feature of the strongly disordered Anderson-Hubbard Hamiltonian is that particle localization occurs independently of the doping level. In the non-disordered case, localization is induced by correlation and gradually disappears as the density moves away from half-filling. Despite this different doping dependence, there are similarities with the ordered case that point to a common localization-related origin of the pseudogap. We found that the pseudogap scale is independent of $U$ for very large $U$ with $A(\omega=\mu)>0$ at any finite doping. To make this point more quantitative we show the $U=8$ and $U=16$ pseudogaps in the left panel of Fig. 3 at the common disorder strength $\Delta=8$. One can see that, apart from a deepening of $A(\omega)$ with increasing $U$, the pseudogap scale is essentially unchanged. Independence of the pseudogap from $U$ and $\Delta$ in the strongly disordered and strongly interacting regime leaves $t$ as the only energy scale relevant to the pseudogap phenomenon. Indeed, halving $t$ (right panel of Fig 3) induces an almost linear reduction on the energy scale characterizing the anomaly. This suggests a kinetic mechanism for pseudo- 

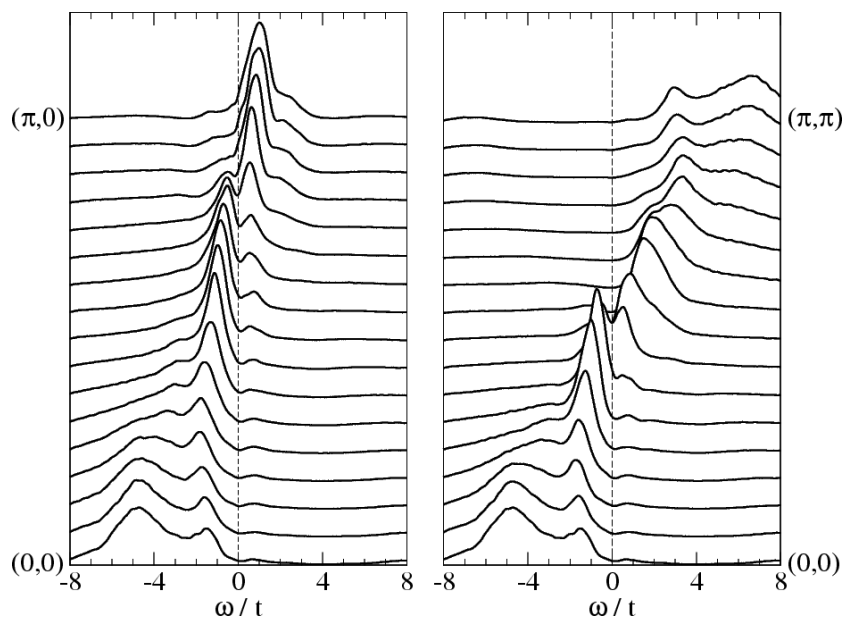

FIG. 4: The momentum resolved spectral function $A_{k}(\omega)$ exhibits a pseudogap as the Fermi surface is crossed. Here $U=8, \Delta=8$, and $\rho=0.8$.

gap formation and it certainly rules out super-exchange since the magnetic energy scale $J$ tends to 0 in this limit. Spin, however, has to play a crucial role since, obviously, spinless fermions interacting through a local $U$ would show a smooth $A(\omega)$ with no suppression at the Fermi surface.

An interesting feature of the clean Hubbard model and of experiments on cuprates is the momentum dependence of the pseudogap. In the hole doped case for example, sharp excitations survive as one crosses the Fermi energy along the nodal direction whereas a pseudogap develops along the anti-nodal one. The behavior of the averaged $A_{k}(\omega)$ in the strongly disordered regime $(\Delta=8)$ is given in Fig. 目. As $k$ cuts through the Fermi surface either along the anti-nodal $((0,0)$ to $(\pi, 0))$ or nodal $((0,0)$ to $(\pi, \pi))$ lines, a depression in $A_{k}(\omega)$ is seen as $k_{F}$ is traversed. This is therefore at odds with the results on the clean model and experimental data. A qualitative explanation for such a difference could reside in the different nature of electron localization in the two cases: while localization is certainly isotropic in the strongly disordered scenario this is not necessarily so in clean materials where it is induced by dynamical inhomogeneities.

In conclusion, we have shown the formation of a robust pseudogap in systems with strong repulsive local interaction and strong disorder. This parameter regime lies outside the range of applicability of the perturbative AA calculation. It also lies outside the Coulomb gap scenario since the potential is local, and there is no Coulomb gap for the on-site Hubbard interaction when the itinerancy of the electrons is switched off.

Although our exact Lanczos analysis is carried out only on clusters small enough to be exactly diagonalized, the phenomenon is also present on much larger clusters treated with the exact DQMC method. The pseudogap energy scale is set by $t$, a result shared with other recent numerical studies of non-disordered, strongly interacting systems [4, 5]. In particular, it persists even when $J \rightarrow 0$, and so does not appear to be linked to antiferromagnetic fluctuations. Finally, it is suggestive that recent experiments [6] observed a doping independent anomalous behavior in the conductivity of highly disordered cuprates consistent with what is reported here.

In the uniform Hubbard model, and the cuprate materials which it may describe, spatial inhomogeneities arise spontaneously, without any explicit symmetry-breaking in the Hamiltonian itself [15, 16]. Likewise, the pseudogap is a feature of the model [3, 4, [5] and the materials. Both phenomena disappear with doping. Disorder however induces i)the stabilization of the pseudogap over a much larger range of densities; ii) its independence on the momentum. These facts, together with the persistence of the pseudogap at large values of $U$ in both disordered and uniform models, are compatible with a scenario where the pseudogap in the strong-coupling regime is intrinsically linked to electron localization and driven by a kinetic mechanism. Such a picture, which invokes spatial inhomogeneities, thus connects the appearance of the pseudogap in the two seemingly rather different contexts of metal-insulator transitions driven by the interplay of randomness and correlation, and the cuprate superconductors.

This research was sponsored by the National Nuclear Security Administration under the Stewardship Science Academic Alliances program through DOE Research Grant DOE DE-FG01-06NA26204. We are grateful for input from J. Garcia, to M. Jarrell for use of his Maximum Entropy code and to R. Singh and G. Zimanyi for many useful discussions.

* Electronic address: chiesa@ucdavis.edu

[1] B.L. Altshuler and A.G. Aronov, Solid State Commun. 30, 115 (1979).

[2] A.L. Efros and B.I. Shklovskii, J. Phys. C8, L49 (1975).

[3] S. Moukouri etal. Phys. Rev. B61, 7887 (2000).

[4] B. Kyung etal, Phys. Rev. Lett. 93, 147004 (2004).

[5] B. Kyung etal, Phys. Rev. B73, 165114 (2006).

[6] S.H. Naqib, J.R. Cooper, R.S. Islam, and J.L. Tallon, Phys. Rev. B71, 184510 (2005).

[7] R. Blankenbecler, D.J. Scalapino, and R.L. Sugar, Phys. Rev. D24, 2278 (1981).

[8] E. Dagotto Rev. Mod. Phys. 66, 763 (1994).

[9] J. Tinka Gammel, D.K. Campbell, E.Y. Loh, cond-mat/9209026

[10] C. Gros, Phys. Rev. B53, 6865 (1996).

[11] R. Haydock, V. Heine, and M.J. Kelly, J. Phys. Chem. 8, 2591, (1975).

[12] J.E. Gubernatis, M. Jarrell, R.N. Silver, and D.S. Sivia, Phys. Rev. B44, 6011 (1991).

[13] Y. Song, R. Wortis, and W.A. Atkinson, Phys. Rev. B77, 54202, (2005).

[14] M. Randeria, R. Sensarma, N. Trivedi, and F-C. Zhang 
Phys. Rev. Lett. 95, 137001, (2005)

[15] K. McElroy et al., Phys. Rev. Lett. 94, 197005 (2005); T. Hanaguri et al., Nature 430, 1001 (2004); M. Vershinin et al., Science 303, 1995, (2004); H. A. Mook, P. Dai, and F. Dogan, Phys. Rev. Lett. 88 , 097004 (2002); J. M. Tranquada et al., Phys. Rev. Lett. 78, 338 (1997).

[16] S.R. White and D.J. Scalapino, Phys. Rev. Lett. 91,
136403 (2003).

[17] Eventually, in the limit $\Delta \rightarrow \infty$, the density of state has to tend uniformly to 0 because of the normalization constraint so that this trend is inverted.

[18] Strong on-site disorder leads to a severe sign problem at half-filling contrary to the $\Delta=0$ situation 